\title{
SOBRE RANKINGS Y ALGO MÁS
}

\author{
Mg. Ebor Fairlie Frisancho* \\ efairlie@gmail.com
}

\section{RESUMEN}

El presente artículo expone las razones por las que las universidades de nuestro país simplemente no figuran en los rankings extranjeros y para esto nos valemos del último ranking del Times Higher Education World University Ranking de Londres, recientemente publicado el 03 de Octubre, con el propósito de poner más desempeño en los rubros y acercarnos más a los estándares fijados a nivel internacional o ir en camino hacia estos.

Palabras clave: ranking universitario, thomson reuters, evaluación de áreas, indicadores de desempeño.

\section{ABSTRACT}

This article outlines the reasons why the universities of our country simply do not figure in the rankings for this foreign and we use the latest rankings of the Times Higher Education World University Ranking of London, recently published on 03 October, with the aim of performance put more items and get closer to the standards set internationally or going on the road to these.

Keywords: University ranking, Thomson Reuters, evaluating áreas, Assessment areas, performance indicators.

\footnotetext{
* Doctorando en Administración, Magister en Administración y Licenciado en Administración de Empresas. Profesor auxiliar e investigador del Instituto de Investigaciones. Asesor de la Oficina de Calidad Académica y Acreditación. Coordinador Académico de la Unidad de Posgrado de la Facultad de Ciencias Administrativas.
} 


\section{INTRODUCCIÓN}

Entre los grupos de estudio que tengo en la red social del Facebook con mis alumnos ingresantes 2012, uno de ellos me comentó acerca de la columna de Andrés Oppenheimer sobre un nuevo ranking internacional en el que no figuraba la Decana de América y que por supuesto no había sido comentado en alguna de las noticias de la universidad, ni en algún diario, revista u otros de nuestro país.

a. Efectivamente, el 06 de octubre de 2012, salió publicado en el Nuevo Herald de Miami, un artículo en la columna El informe Oppenheimer y cuyo título dice "Malas noticias para universidades latinoamericanas".

b. EL artículo menciona entre otras cosas lo siguiente:

c. Ha salido un nuevo ranking internacional de las mejores universidades latinoamericanas realizado por Thomson Reuters el último 03 de Octubre el cual ha pasado casi desapercibido por la población mundial dado por ejemplo el mayor interés que tenían las elecciones en Venezuela.

d. En dicho ranking, se menciona que no hay ninguna universidad latinoamericana en el top 100 y apenas solo cuatro Universidades latinoamericanas figuran entre las 400 mejores, siendo Brasil y México las mejores economías del mundo. Del resto no hay signos de existencia.

e. Las cuatro universidades latinoamericanas que figuran en dicho ranking son la Universidad Sao Paulo y la universidad Estatal de Campiñas de Brasil, la Universidad de los Andes de Colombia y la Universidad Nacional Autónoma de México.

f. Existen 22 universidades asiáticas entre las 200 mejores del mundo y 56 instituciones asiáticas entre las mejores 400 del mundo, que dicho sea de paso, están ascendiendo con gran rapidez en todos los rankings.

g. Para variar, las universidades estadounidenses ocupan los primeros puestos, de las cuales siete de las 10 primeras son de Estados Unidos y que poco a poco están siendo desplazadas por Universidades asiáticas.

h. Adicionalmente indica también que ni el QS World University Ranking de Londres ni el de la universidad de Shanghai tiene entre las 100 primeras instituciones a universidades latinoamericanas.

i. Menciona además que muchas de las universidades extranjeras como de Estados Unidos y Corea del Sur invierten el 2.6\% del PBI en investigación tanto como México y Argentina.

j. Una de las razones que indica de porqué en las universidades latinoamericanas no se hace la inversión en investigación avanzada es el alto número de estudiantes y, además, le ponen mayor prioridad a los gastos de infraestructura.

k. Comenta también que muchos gobiernos latinoamericanos objetan muchos rankings extranjeros porque no están acordes con la realidad latinoamericana y que tienden a favorecer a países de habla inglesa. Incluso dicen que debería haber un ranking exclusivo para países latinoamericanos.

1. El autor opina que es totalmente falso que el idioma sea una excusa para no figurar en estos rankings y que tener un ranking regional hecho a medida de las Universidades latinoamericanas "son recetas para la autocomplacencia, la parálisis y el atraso".

Este es el resumen del cual vamos a partir para conocer la forma de calificación del Times Higher Education World University Ranking de Thomson Reuters y observar porque la Universidad Nacional Mayor de San Marcos se encuentra muy lejos de cumplir los mínimos requisitos de dicho ranking y por ende de otros internacionales.

\section{ANÁLISIS CONCEPTUAL}

Thomson Reuters es una empresa de información, creada por la compra de Reuters por la Corporación The Thomson Corporation el 17 de abril del 2008. Las acciones de Thomson Reuters cotizan en la Bolsa de Valores de Toronto (TSX: TRI) y la Bolsa de Nueva York (NYSE: TRI). La sede operativa se encuentra en el Midtown Manhattan, Nueva York. Thomson Reuters opera en 93 países, y cuenta con más de 50.000 empleados, (Wikipedia, 2012).

Thomson Reuters es una institución reconocida de alto prestigio académico que además de ofrecer servicios de información, tiene lo que a mi particular parecer indica, una de las más reconocidas y prestigiosas bases de datos de 
información académica disponible que usan muchas Universidades: el famoso Institute Scientific Information Web of Knowledge, adicional a que el portal de Bibliotecas de nuestra Universidad indica "El Current Contents Connect del ISI (Institute for Scientific Information) - Instituto para la Información Científica - es un recurso actual multidisciplinario del ISI Web que proporciona acceso a información bibliográfica de cerca de 8,000 revistas académicas más reciente y evaluados por expertos." (UNMSM, portal de biblioteca, 2012).

The Times Higher Education World University Ranking es un ranking elaborado por Thomson Reuters que se encuentra reconocida a nivel mundial por la seriedad de sus publicaciones que ha demostrado año tras año ser un referente para las comparaciones respectivas entre los avances logrados de todas las Universidades participantes.

El encargado y editor de dicho ranking es Phil Baty, en cuya biografía aparece su trayectoria profesional en el periodismo, ganador del premio Ted Wragg por una contribución sostenida para la educación en periodismo el 2011, y el mismo año bajo su mando, la revista Times Higher Education fue nombrada la revista semanal y marca del año en la categoría de negocios por la asociación de publicistas profesionales. The Australian Newspaper considera a Phil uno de los quince mejores personajes que más influencia tienen en educación. (Times Higher Education, Biografía Phil Baty, 2012).

\section{SOBRE EL RANKING}

El Times Higher Education World University Ranking 2012-2013 auspiciado por Thomson Reuters "representan las únicas tablas de rendimiento global universitario para calificar a las universidades de clase mundial a través de sus misiones centrales como son la docencia, investigación, transferencia de conocimiento y la proyección internacional". Este ranking utiliza 13 indicadores de desempeño para efectuar las comparaciones más completas y proporcionadas que son aceptadas por los estudiantes, académicos, líderes universitarios, la industria y los gobiernos (Times Higher Education, 2012).

Estos indicadores están acorde a los 8 factores que la Universidad Nacional Mayor de San Marcos tiene acorde al SINEACE y al modelo de autoevaluación y acreditación que nos encontramos aplicando en muchas Facultades y en donde intervienen los académicos, administrativos, alumnos, empresarios y la comunidad. Tarde o temprano vamos a tener que llegar a entrar en estos rankings dado que tienen mucho parecido, pero recién estamos empezando a pasos lentos. Nos falta mucho tiempo.

\section{OPINIÓN DE VARIOS EXPERTOS SOBRE EL RANKING}

En la misma página del Times Higher Education, existen varias opiniones de editores y diversas personalidades sobre el ranking con explicaciones y experiencias fundamentando el estudio realizado por THE. Entre ellos tenemos los siguientes:

- Phil Baty considera el ranking una gran responsabilidad y que es reconocido a nivel internacional y es tomado en cuenta no solamente por muchos países, sino por los futuros alumnos. Dice "Esto no es un desfile de belleza, es una evaluación seria que resuena en las salas comunes y los pasillos del poder". Analiza también los Top 200 indicando "El poder es donde el poder va”. Lo completa con un análisis entre los 200 y 400 del resto de la lista, hace un resumen de los que "eran" gigantes y empiezan a descender. Por último y con lo que también estoy de acuerdo, la reputación de una universidad es el factor más importante para la mayoría de los potenciales estudiantes internacionales.

- Dirk Van Damme descifra las tendencias cambiantes de la educación superior universitaria a nivel mundial argumentando que no hay complot contra las "nuevas" Universidades.

- David Willetts, Ministro de las Universidades y de la Ciencia de Inglaterra, menciona que hay que mantenerse en movimiento y que no hay lugar para la complacencia.

- Bernd Huber explica que la rivalidad es vital para el desarrollo de las instituciones de clase mundial tornándose a futuro en una ventaja competitiva.

- Ed Byrne recomienda la colaboración internacional entre las instituciones para lograr la excelencia y que tengan la famosa fórmula Win-Win.

- De la Universidad de Stanford, John Hennessy argumenta que este ranking es la mejor opción, ya que el e-Iearning ofrece un camino a 
seguir por las Universidades occidentales, es decir enseñanzas virtuales.

- Max Precio precisa que es vital la labor de investigación en los países en desarrollo como el nuestro, pero paso por paso, localmente, globalmente y no ser cola de león.

- Bertil Anderson habla de Singapur que ha hecho inversiones sustanciales en investigación con colaboración académica internacional ayudando a que crezca rápidamente y se encuentre considerada en este ranking.

- Karan Khemba menciona que el ranking se encuentra dominado por Universidades Occidentales, pero los mercados emergentes y las nuevas Universidades siguen aumentando.

- Por último, las opiniones de Stephen Crookbain y Bottomley consideran los pro y los contra de estar o no en este ranking y los beneficios para la Institución.

\section{MÉTODO}

El Times Higher Education utiliza el mismo criterio del año 2011, en donde son 13 los indicadores de desempeño agrupadas en cinco áreas. La metodología no ha cambiado para hacer comparaciones año tras año y ver la evolución de las Universidades que se quedan.

Las áreas son

» Enseñanza: el ambiente de aprendizaje (30\% de la puntuación de ranking general)

» Investigación: volumen, los ingresos y el prestigio (30\% del ranking)

» Citas: influencia de la investigación $(30 \%$ del ranking)

» Ingresos de la industria: innovación (2.5\% del ranking)

» Perspectiva internacional: académicos, estudiantes y la investigación $(7.5 \%)$.

\section{EXCLUSIONES}

Algunas Universidades no han sido consideradas en este ranking por:

» No enseñar carreras de pregrado

» Sólo enseñan una simple área o tema

» Si las investigaciones publicadas suman menos de mil artículos entre los años 2006 al

\section{0 (200 por año)}

"En algunos casos excepcionales se han considerado instituciones que han publicado menos de 200 artículos, si y solo si se enfocaron en disciplinas con poca publicación de volúmenes como ingeniería, artes o humanidades.

\section{PUNTUACIÓN}

Para el cálculo de la clasificación del ranking, "puntajes Z" fueron creados para todo el conjunto de datos a excepción de los resultados de la encuesta de prestigio académico. El cálculo de estos puntajes se estandariza los diferentes tipos de datos en una escala común y permite comparaciones justas entre los diferentes tipos de datos esenciales al combinar información diversa en una clasificación única para que sea justa para todos los evaluados.

A cada punto de datos se le da una puntuación basada en su distancia de la media del conjunto de datos completos donde la escala es la desviación estándar del conjunto de datos. Este puntaje es convertido en una "puntuación de probabilidad acumulada" para llegar a los totales finales.

\section{OBTENCIÓN DE DATOS}

Las Instituciones analizadas brindan datos e información para ser utilizados en el ranking, es decir se pide la colaboración de las instituciones involucradas.

En raras ocasiones cuando algún dato en particular no se muestra y afecta los indicadores más bajos como ingresos de la industria, por ejemplo, se coloca el estimado más bajo entre el promedio de valores de los indicadores, evitando penalizar a cualquier Institución con un cero en datos que no se observan o no fueron informados.

\section{ÁREAS A EVALUAR \\ PERSPECTIVA INTERNACIONAL: PERSONAS, IN- VESTIGACIÓN (7.5\%)}

Esta categoría analiza la diversidad en la institución y en qué grado los profesores colaboran con colegas internacionales en proyectos de investigación - ambos signos de la forma global de una institución tiene perspectiva internacional. Esta categoría se compone de tres indicadores:

a. La capacidad de la universidad para atraer a estudiantes de pre y posgrado de diferentes países es clave para su éxito en el escenario 
mundial: este factor se mide por la relación de estudiantes internacionales y es calificado con el $2.5 \%$ de la puntuación total.

b. Las mejores universidades también compiten por tener los mejores profesores de todo el mundo. Así que en esta categoría se coloca $2.5 \%$ de ponderación de la relación internacional de profesores.

c. En el tercer indicador internacional, se calcula la proporción del total de publicaciones de revista de investigación que tienen por lo menos un co-autor internacional y compensar grandes colecciones equivalente a $2.5 \%$ de esta puntuación.

\section{INVESTIGACIÓN: VOLUMEN, INGRESOS, PRESTI- GIO (30\%)}

Esta categoría se compone de tres indicadores. El más importante con un $18 \%$, analiza el prestigio de una universidad para la excelencia en investigación entre sus pares basado en las 16,000 respuestas de la encuesta anual de prestigio académico realizada por el Times Higher Education todos los años.

Esta categoría también analiza los ingresos obtenidos por investigación universitaria, contrastados con el número de investigadores, categoría muy discutida porque puede ser influenciado por la política nacional y las circunstancias económicas del entorno y país.

Desafortunadamente (y en lo que también estoy de acuerdo), los ingresos son cruciales para el desarrollo de la investigación de clase mundial y porque gran parte de ella está sometida a la competencia y calificada mediante evaluación por pares, los expertos de Times Higher Education indicaron que es una medida válida.

Este indicador está totalmente normalizado para tener en cuenta el perfil de cada área del conocimiento de cada universidad, lo que refleja el hecho de que las becas de investigación en áreas científicas son mayores que los otorgados a las áreas de ciencias sociales, artes y humanidades. Se le da una ponderación de $6 \%$

La categoría de investigación también incluye una medida simple de la productividad de la investigación - resultados de la investigación versus el número de profesores. Contamos el número de artículos publicados en las revistas científicas indexadas por Thomson Reuters de cada institu- ción académica, en una escala para el tamaño total de una universidad y normalizada por área. Esto da una idea de la capacidad de una institución para obtener trabajos publicados en revistas de calidad revisadas por pares. Aquí le otorgan un $6 \%$ en general.

\section{CITAS: INFLUENCIA DE LA INVESTIGACIÓN (30\%)}

Nuestro indicador de la influencia de la investigación es el caballo de batalla de este ranking. Ponderado en el $30 \%$ de la puntuación total, es el más influyente de los 13 indicadores, y examina el papel de las universidades en la difusión de nuevos conocimientos e ideas.

Se examina la influencia de la investigación acorde al número de veces que se cita un trabajo publicado por investigadores (docentes o alumnos) de la Universidad a nivel mundial. Para este ranking, Thomson Reuters ha examinado más de 50 millones de citas a 6 millones de artículos de revistas, publicados en cinco años. Los datos se han extraído de las 12.000 revistas académicas indexadas por la base de datos "Web of Science" de Thomson Reuters incluyendo todas las revistas indexadas publicadas entre el 2006 y 2010.

Las citas nos ayudan a mostrar de qué forma cada universidad está contribuyendo a la suma del conocimiento: nos dicen qué investigación ha destacado, si ha sido recogida y aumentada por otros investigadores y, lo más importante, si ha sido compartida por la comunidad científica mundial para superar los límites de la comprensión colectiva, independientemente del área en que se desarrolla la investigación.

Los datos se normalizaron por completo para reflejar las variaciones en la cantidad de citas entre diferentes áreas temáticas. Esto significa que las instituciones con altos niveles de actividad de investigación en áreas con alto número de citas no ganarían como siempre una ventaja injusta vs. otras áreas que no tienen un gran número de citas.

No se incluye en este ranking alguna institución que publica menos de 200 artículos al año para asegurar comparaciones de datos estadísticamente válidos.

\section{INGRESOS DE LA INDUSTRIA: INNOVACIÓN (2.5\%)}

La capacidad de una Universidad para ayudar a la industria con inventos, innovaciones y consultorías se ha tornado la misión principal de las 
instituciones de clase mundial. Se busca pues identificar la transferencia tecnológica identificando los ingresos por investigación que gana de la industria, acorde al número de profesores que participen.

Este indicador sugiere en que negocios centrarnos y que negocios son factibles que paguen por la investigación y que la Universidad tenga la capacidad de atraer inversiones competitivas en el mercado comercial, los que usualmente indican la calidad de la institución. Esta categoría esta con un $2.5 \%$ del ranking.

\section{ENSEÑANZA: EL AMBIENTE DE APRENDIZAJE $(30 \%)$}

Esta categoría cuenta con cinco indicadores de desempeño diseñados para proporcionar una visión clara de la enseñanza y el entorno de aprendizaje de cada institución tomando en cuenta la perspectiva del estudiante y los profesores.

El principal indicador utiliza los resultados de la encuesta de prestigio (sólo por invitación) más grande del mundo, tomada en Abril 2012 y cuya impulsadora es Thomson Reuters a académicos con experiencia, examinando el prestigio percibido de las instituciones tanto en la investigación y la docencia con un total de 16,639 respuestas estadísticamente representativas a nivel geográfico y por áreas de la educación superior a nivel mundial. A estos resultados se les da el $15 \%$ de la puntuación total.

La categoría de la enseñanza y el aprendizaje también emplea un ratio profesor-estudiante (número total de estudiantes de una institución) como un simple (y lamentable) indicador de calidad de la enseñanza.

El indicador sugiere que donde hay una proporción saludable entre estudiantes y profesores, los primeros recibirán la atención personalizada que requieren de los profesores de la institución. Esta ponderada con un $4.5 \%$ de la clasificación general.

La categoría de enseñanza examina la relación de grados de Bachiller a Doctor otorgadas por cada institución.

Creemos que instituciones con una alta densidad de estudiantes de investigación son más intensivos en conocimiento y que la presencia de una comunidad activa de posgrado es un marcador de una investigación dirigida por el entorno de enseñanza valorado por los estudiantes y posgra- duados. A esta relación le corresponde $2.25 \%$ de la puntuación.

La categoría docente también utiliza datos sobre el número de grados de Doctor otorgados por una institución comparado con el número de profesores que enseñan. Esto compromete a una institución para crear la siguiente generación de profesores, es por eso que una alta proporción de estudiantes de investigación de posgrado indica la previsión de enseñanza al más alto nivel y que sea atractivo para los graduados de tal manera que continúen en la institución de origen.

Los estudiantes de pregrado también tienden a valorar el trabajar con estudiantes de posgrado. Este indicador está normalizado para tener en cuenta todas las áreas de una Universidad, reflejado en la cantidad de Doctorados en diferentes disciplinas, representando el $6 \%$ de la puntuación.

El indicador final en esta categoría es una medida simple de los ingresos de la institución versus el número de profesores. Esta cifra, ajustada para que todos los países puedan competir en igualdad de condiciones, indica el estado general de la institución entre la infraestructura y las instalaciones disponibles para los estudiantes y los profesores. Tiene un valor $2.25 \%$ en general.

\section{RESULTADOS Y DISCUSIÓN}

Efectuaremos una comparación rápida entre lo que se indica en el ranking vs. lo que sucede en la Universidad Nacional Mayor de San Marcos desde los requisitos hasta cada categoría e indicador acorde a los datos hallados en nuestra Universidad.

\section{REQUISITOS}

» Se enseñan carreras de pregrado. CUMPLE

» Tenemos más de 60 carreras o especialidades. CUMPLE

" "Si las investigaciones publicadas suman menos de mil artículos entre los años 2006 al 2010 (200 por año)”. Acá si hay una seria duda entre el cumplimiento de este requisito. No existen datos en la página web de la Universidad a la fecha, pero la última estadística de publicación que aparece en la página web del Consejo Superior de Investigaciones del vicerrectorado del año 2006, indica que se han publicado solo 23 artículos en el ISI Web of Knowledge de Thomson Reuters. No podemos 
extrapolar este resultado para explorar qué tan bien nos fue del año 2007 al 2010 y que podamos publicar el resto de los 977 artículos publicados en esos años. Lo veo casi improbable y existe una gran posibilidad que no se haya tomado en cuenta y no aparezcamos en este ranking por este motivo.

"En algunos casos excepcionales se han considerado instituciones que han publicado menos de 200 artículos". Como es seguro que estemos en este rubro porque nuestra Universidad más se orienta a carreras de Ciencias Sociales u otros, no he visto ni en la imprenta o editorial de la Universidad grandes volúmenes de libros o artículos que puedan estar en esta categoría en ninguna Facultad durante esos años $\mathrm{y} / \mathrm{o}$ al menos no se han publicado registros de este tipo.

Respecto a las categorías, tenemos las siguientes relaciones:

1. Enseñanza: el ambiente de aprendizaje (30 \% del ranking)

Con respecto al primer indicador, encontramos que no hemos participado de la encuesta de Abril de 2012 de Thomson Reuters, por lo que no tenemos ya un $15 \%$. Respecto al segundo indicador, acorde a las últimas estadísticas de matrícula de la página web del SUM, tenemos un total de 30230 alumnos matriculados el 2012-I con un aproximado de 3000 docentes, de los cuales aproximadamente la tercera parte se encuentran solo en la Facultad de Medicina, pero solo un $8.5 \%$ de los matriculados dejando un promedio de 2000 profesores para 27671 alumnos en general cosa q parece un promedio de 13 o 14 alumnos por profesor, pero eso no es general; en la Facultad por ejemplo es común ver entre 45 o 75 alumnos por profesor/ curso, cosa impensable en cursos de tesis o investigación.

El tercer indicador menciona un ratio de grados otorgados entre Bachiller y Doctor. Entre Julio 2011 y Julio 2012 se han otorgado 71 grados de Doctor a comparación de un aproximado de 3000 grados de Bachiller anuales es un rango bastante grande. Adicionalmente que en la práctica un mínimo de todos los Doctores que se gradúan tienen presencia en el entorno de enseñanza de pregrado a menos que se gradue un profesor.
El cuarto indicador demuestra cuantos profesores tienen el grado de Doctor y que enseñan en la Universidad; esto es un poco difícil de obtener, dado que no todos los profesores estamos obligados a decir cuando obtenemos el grado de Doctor ni informar al respecto; en la Facultad por ejemplo, de los 116 profesores nombrados son 20 los que tienen registrados su Doctorado. En otras Facultades el rango es mayor dado que están acreditadas. Por último el indicador final de ingresos vs. la cantidad de profesores en donde vemos que se siguen creando nuevas carreras, es cierto que estamos homologados en parte con el poder judicial, pero los ingresos dependen de la importancia que se le da a la marca Universidad y la distribución para considerar la importancia a los profesores.

2. Investigación: volumen, los ingresos y la prestigio ( $30 \%$ del ranking)

Respecto a este rubro tenemos tres indicadores. El primero sobre el prestigio de la Universidad de la encuesta tomada por Times Higher Education de la que no hemos participado. Otro es sobre los ingresos de la Universidad vs. el número de investigadores que en el caso de nuestra Facultad de los 116 profesores, oficialmente son 21 profesores los que cumplen los requisitos para ser investigadores pero los ingresos no son distribuidos para el desarrollo de la investigación y ni que hablar con la cantidad de becas de investigación. Respecto a los resultados de investigación vs. el número de profesores, en muchos de los casos, las investigaciones no se convierten en artículos y mucho menos publicados en revistas científicas publicadas por Thomson Reuters considerando que nuestra revista no esta dentro de ese tipo de revistas asi como otras facultades.

3. Citas: influencia de la investigación $(30 \% \mathrm{del}$ ranking)

" De hecho que en este rubro no estamos considerados, ya que no se toman en cuenta aquellas Universidades que no hayan hecho al menos 200 publicaciones al año entre el 2006 y 2010 . Ya no tener una tercera parte del puntaje es una gran desventaja. Adicionalmente que para nuestro caso, la revista de nuestra Facultad (y la de casi la mayoría de las Facultades) no esta indexada por la base de datos de Thomson Reuters. 
4. Ingresos de la industria: innovación $(2.5 \% \mathrm{del}$ ranking)

En este indicador hablan de centrarnos en negocios que tengan la capacidad de atraer inversiones en el mercado comercial y crear inventos o consultorías. Esto lo usan bastante en otras Facultades como Medicina, psicología, odontología, pero al menos el área de ciencias empresariales no tiene centros de producción para poder tener ingresos y ser referente en el Perú como Universidad. Esto lo vemos solamente con investigaciones personales o tesis que algunos proponen, pero no se ven los ingresos por la solución propuesta y por lo tanto no hay ingresos para los profesores que lo proponen.

5. Perspectiva internacional: académicos, estudiantes y la investigación (7.5\%).

» $\mathrm{Si}$ se considera nuestra capacidad de atraer estudiantes de pregrado, la imagen de nuestra Universidad es reconocida a nivel mundial, al menos a lo largo de 3 países latinoamericanos a los que he viajado y muchos desearían estudiar en la Decana de América. Lamentablemente, por ser Universidad pública, no ofrecemos lo que exactamente se dice "vacantes" o becas a nivel mundial, sino que todo el que cumple los requisitos de admisión, puede postular para ser alumno regular. Inclusive en Posgrado de muchas Facultades existen estudiantes internacionales; por ejemplo en nuestra Facultad hemos tenido estudiantes internacionales, 2 o 3 entre 150 alumnos matriculados. Con esto no estamos diciendo que exactamente vengan a estudiar al Perú los 2 años siendo residentes en otro país. Este porcentaje es muy bajo.

» Respecto a los profesores internacionales, existe poca presencia de profesores internacionales en las facultades, ya que más del 95\% son de Perú y no vienen exclusivamente a dictar algún curso. En caso de posgrado por ejemplo y de otras Facultades, se efectúa un convenio con otras Universidades para que varios profesores del extranjero dicten clases en un programa determinado, por ejemplo en el caso de un programa de comercio internacional, de los 16 cursos que se tenía, 6 dictaban profesores franceses que venían a dictar exclusivamente los cursos y 10 profesores nacionales pero sólo en ese programa que a la fecha no esta vigente. Aun así el porcentaje es muy bajo en comparación con Universidades americanas en el que tienen al $40 \%$ del plantel internacional en todos sus programas.

» Respecto a la revista de investigación que sólo se tiene en pregrado, observo en varias revistas que si hay un interesante número de artículos con coautores de diversos países internacionales en especial de las áreas como medicina, enfermería.

\section{CONCLUSIONES}

1. A pesar de estar primeros en el "Ranking mundial de Universidades en la web" en nuestro país y estar considerados en primer lugar dentro de las mil Universidades en el puesto 810 (Secretaría General de la UNMSM), estamos muy por debajo de estar entre las 500 mejores del mundo que al menos reconocen la mayoría de los rankings internacionales.

2. El ranking de Times Higher Education es reconocido a nivel mundial tanto en Estados Unidos y Europa y por lo tanto considero que nuestra Universidad que esta acreditando carreras, tiene que tomarlo en cuenta.

3. La universidad ya se está enfocando en estos mercados internacionales, por lo que es necesario cumplir como Universidad del futuro y modelo latinoamericano los parámetros que son requeridos por este ranking.

4. Es cierto que la universidad está pasando por un proceso de acreditación del SINEACE desde la autoevaluación hasta la acreditación en todas sus fases. Tenemos que avanzar raudamente para poder estar cerca de estos rankings, de otra forma no estaremos considerados a nivel internacional.

5. No podemos quedarnos en la acreditación del SINEACE y esperar aparecer, por ejemplo, en este ranking. Tenemos que seguir los nuevos estándares y competir por ser los mejores. No por algo tenemos 461 años.

6. Aun estamos muy por debajo de lo mínimo requerido por este ranking con respecto a su admisión, es decir los artículos de investigación que no tenemos. Hay que fomentar entre nuestros docentes y alumnos concursos, premios, publicaciones.

7. Nuestra facultad esta empezando con la iniciativa de crear una revista de investigación 
para posgrado y para los alumnos a través de un grupo estudiantil. Esta iniciativa la estamos haciendo con nuestros propios recursos, pero existen Facultades que no tienen recursos y que la Universidad, el estado, otras instituciones del Estado o empresas deben de apoyar. No es posible que por falta de dinero no se puedan generar publicaciones.

8. Hasta el mes de octubre de 2012, Times Higher Education solicitó para el próximo ranking un contacto para poder participar, pero al parecer aún no cumplimos los requisitos mínimos de los 200 artículos anuales. Esto era solamente para participar en las encuestas de 12,500 revistas de investigación universitarias que tenían 53 millones de artículos individuales.

9. Considero que hay que empezar al menos en nuestra Facultad cumpliendo varios de los requisitos propuestos en este artículo y velar porque lo que las autoridades también lo hagan extensivo a las demás carreras para que todas las Facultades puedan sobresalir en investigación con el apoyo del Vicerrectorado de investigaciones.

\section{LITERATURA CITADA}

Consejo Superior de Investigaciones del Vicerectorado de Investigaciones de la UNMSM (2012). Perú. Disponible el 15 de Junio de 2012). http://vrinvestigacion.unmsm.edu. pe/consejo_superior/publicaciones_registradas/PublicPtje/D_09.pdf

El Nuevo Herald (2012). Estados Unidos. Disponible el 06 de Octubre de 2012. http://www. elnuevoherald.com/2012/10/06/1316638/ malas-noticias-para-universidades.html

Escuela de Posgrado (2012). Grados académicos otorgados. Lima. Disponible Setiembre 2012. http://epg.unmsm.edu.pe/webepg/ index.php?option $=$ com_content\&view $=$ category\&id $=8 \&$ Itemid $=85$.

Estadísticas del Sistema Unico de Matrícula de la Universidad Nacional Mayor de San Marcos (2012). Perú. Disponible el 06 de Octubre de 2012. http://sum.unmsm.edu.pe/estadistica2007.htm.

Base de datos del Sistema de bibliotecas de la UNMSM (2012). Perú. Disponible desde el 06 de Octubre de 2012. http://sisbib. unmsm.edu.pe/pub_electron/base_datos. htm\# isicurrent

Secretaría General de la Universidad Nacional Mayor de San Marcos (2012). Disponible Junio 2012. http://secgen.unmsm.edu.pe/index. php/unmsm-primera-del-pais-en-rankingweb-de-universidades

Times Higher Education (2012). Reino Unido. Disponible el 05 de Octubre de 2012. http:// www.timeshighereducation.co.uk

Times Higher Education World University Rankings (2012). Reino Unido. Disponible el 05 de Octubre de 2012. http://www.timeshighereducation.co.uk/world-universityrankings.

Wikipedia (2012). España. Disponible el 9 de Agosto de 2012. http://es.wikipedia.org/ wiki/Thomson_Reuters 